\title{
PD-L1 Expression Enhancement by Tumor- Associated Macrophages Derived Osteopontin Leads to Poor Non-Small Cell Lung Caner Prognosis
}

Yue Li

Department of Lung Cancer,Tianjin Medical University Cancer Institute and Hospital,National Clinical Research Center for Cancer,Key Laboratory of Cancer Prevention and Therapy,Tianjin's Clinical Research Center for Cancer,Tianjin Lung Cancer Center

Hai-lin Liu

Department of Lung Cancer,Tianjin Medical University Cancer Institute and Hospital,National Clinical Research Center for Cancer,Key Laboratory of Cancer Prevention and Therapy,Tianjin's Clinical Research Center for Cancer,Tianjin Lung Cancer Center

\section{Dong-sheng Yue}

Department of Lung Cancer,Tianjin Medical University Cancer Institute and Hospital, National Clinical Research Center for Cancer,Key Laboratory of Cancer Prevention and Therapy,Tianjin's Clinical Research Center for Cancer,Tianjin Lung Cancer Center

\section{Chen Chen}

Department of Lung Cancer,Tianjin Medical University Cancer Institute and Hospital,National Clinical Research Center for Cancer,Key Laboratory of Cancer Prevention and Therapy,Tianjin's Clinical Research Center for Cancer,Tianjin Lung Cancer Center

\section{Chen-guang Li}

Department of Lung Cancer,Tianjin Medical University Cancer Institute and Hospital,National Clinical Research Center for Cancer,Key Laboratory of Cancer Prevention and Therapy,Tianjin's Clinical Research Center for Cancer,Tianjin Lung Cancer Center

\section{Zhen-fa Zhang}

Department of Lung Cancer,Tianjin Medical University Cancer Institute and Hospital,National Clinical Research Center for Cancer,Key Laboratory of Cancer Prevention and Therapy,Tianjin's Clinical Research Center for Cancer,Tianjin Lung Cancer Center

\section{Sheng-qiang $\mathrm{Xu}$}

YuceBio Technology Co.,Ltd.,Shenzhen

Hui-ping Lu

YuceBio Technology Co.,Ltd.,Shenzhen

\section{Tian-tian Gu}

YuceBio Technology Co., Ltd.,Shenzhen

Chang-li Wang ( $\sim$ lab_2020@126.com ) 
Department of Lung Cancer,Tianjin Cancer Institute and Hospital,Tianjin Medical University

\section{Research}

Keywords: Lung cancer, Macrophages, Osteopontin, PD-L1

Posted Date: September 28th, 2020

DOI: https://doi.org/10.21203/rs.3.rs-66234/v1

License: (c) (i) This work is licensed under a Creative Commons Attribution 4.0 International License. Read Full License 


\section{Abstract}

\section{Background}

In the tumor microenvironment, programmed death ligand 1(PD-L1) is a key protein upregulated by tumor cells in immune escape. Tumor-associated macrophages (TAMs) play a major role in this immunosuppression. Osteopontin (OPN) is related to tumor metastasis and proliferation and immunosuppression. OPN is expressed not only by tumor cells, but also by TAMs. However, little is known of the relationship between OPN expressed by TAMs(TOPN) and PD-L1 in non-small cell lung cancer.

\section{Methods}

Tissue microarray was used to detect the expression of TOPN, TAMs and PD-L1 by multiple quantitative fluorescence staining in 509 NSCLC patients undergoing complete pulmonary resection. The correlations between TOPN, PD-L1 and clinicopathologic data were analyzed. And in vitro cell cocultures were further conducted to investigate the crosstalk between TOPN and Neoplastic PD-L1. The in vivo efficacy of TOPN was evaluated for PD-L1 expression in subcutaneous coculture mouse model of NSCLC.

\section{Results}

We observed a positive association between the TOPN and PD-L1 expression in tumor tissues from 509 patients with NSCLC. In addition, survival analysis revealed that TOPN and PD-L1 were independent prognostic factors for overall survival(OS) and disease-free survival(DFS) of NSCLC patients. We further demonstrated that TOPN upregulated PD-L1 expression in NSCLC cells through NF-KB pathway in vitro. And the upregulation was inhibited by neutralization with anti-OPN antibody or NF-KB inhibitor after coculture with macrophages. TOPN induced the PD-L1 expression in tumor-bearing mice, and promoted the tumor growth.

\section{Conclusions}

TOPN/NF-KB axis plays a critical role in PD-L1 expression of NSCLC microenvironment. TOPN could be a potential therapeutic target for NSCLC.

\section{Background}

Lung cancer remains the most lethal human malignancy ${ }^{1}$. Non-small cell lung cancer (NSCLC) comprises approximately $80 \%$ of all cases ${ }^{2}$. Only a small number of patients with NSCLC are eligible for receiving curative treatment, such as complete pulmonary resection and systematic lymph node dissection. Amount of NSCLC patients were at the risk of a high rate of distant metastasis and local recurrence after resection ${ }^{3}$. Although the therapy, including chemotherapy, radiotherapy and molecule-targeted treatment, is more improved, the mortality rate is still high ${ }^{4}$, the overall 5 -year survival rate is only $15 \%{ }^{5}$. Moreover, the US Food and Drug Administration (FDA) approved anti-programmed death receptor-1 (anti-PD-1) 
antibody nivolumab and pembrolizumab for treatment of advanced-NSCLC ${ }^{6}$. Even though, great improvement in survival has been achieved in a small part of NSCLC patients ${ }^{7}$, many patients could not benefit from 10 therapy ${ }^{8,9}$. Thus, identifying novel therapies to improve long-term patients' outcome are urgently needed.

During the past decade, advances in immunotherapy has transformed cancer treatment by blocking immune checkpoints, for example, programmed death ligand 1 (PD-L1), programmed death receptor 1 (PD-L1) to unleash anti-tumor immune response. Monoclonal antibodies (mAbs) such as nivolumab and pembrolizumab, which target PD-1 have exhibited remarkable clinical responses in a broad spectrum of cancer patients, including NSCLC, melanoma, Hodgkin lymphoma, renal cell carcinoma (RCC), head and neck squamous cell carcinoma ${ }^{9,10}$. PD-L1 is one of the two receptors of PD-1, which can be expressed on the surface of tumor cells, macrophages, B cells. The ligation of PD-L1 on tumor cells and PD-1 of T cells induces $T$ cells lose activity, which leads to further immunosuppression ${ }^{11}$. In tumor cell, PD-L1 overexpression indicates the worsening of NSCLC and poor prognosis ${ }^{12,13}$. Tumors that arise in immunosufficient hosts usually have poor immunogenicity, which is caused by immunoediting. Immune editing, which is a dynamic process of immunosurveillance to immune escape, is influenced by many factors, among which immune microenvironment is the dominant one. In immune microenvironment, tumor associated macrophages (TAMs) play an important role in immunosuppression ${ }^{14-16}$.

Osteopontin(OPN) is a multifunctional glycoprotein ${ }^{17}$. Previous studies by our group ${ }^{18-21}$ and others ${ }^{22}$ demonstrated that OPN, which was an important tumour-sustaining inflammatory mediator, expressed by not only by tumor cells but also by cells in tumor extracellular matrix, such as macrophages, were associated with progression, metastasis and immunosuppression. However, the relationship between TOPN and the expression of PD-L1 in NSCLC microennvironment remains unclear.

\section{Materials And Methods}

\section{Patients}

All NSCLC patients undergoing complete pulmonary resection and systematic lymph node dissection at the Cancer Institute and Hospital of Tianjin Medical University (China) between 2004 and 2012 were considered eligible for retrospective analysis of clinical prognostic factors. Patients who were included in the study met the following criteria: (a) a distinctive NSCLC diagnosis based on World Health Organization criteria; (b) The patients underwent CT or MRI scan for brain, chest and abdomen ,and ECT(Emission Computed Tomography) for bone preoperatively to make sure that there was no metastasis; (c) no prior anticancer treatment;(d) the stage II and IIla patients received adjuvant chemotherapy or radiotherapy, alone or in combination after surgery;(e) complete pulmonary resection and systematic lymph node dissection;(f) complete clinicopathological and follow-up data. Ethical approval for human subjects was obtained from the research ethics committee of Tianjin Cancer Institute and Hospital, and informed consent was obtained from each patient. Patients who died within 1 month 
after surgery were excluded from the study. Tumor staging was based on the most recent IASLC TNM classification system ${ }^{4}$. All patients were followed up until Aug 31, 2019. Patients who were still alive after the last follow-up were censored in the study.

\section{Tissue Microarray and Multiple Quantitative Fluorescence Staining}

Tissue were used to construct a tissue microarray (TMA), as described preciously ${ }^{18}$. To validate the concordance between TMAs and whole tumor sections, we further detected OPN, PD-L1 and CD68 expression for 50 cases randomly chosen from the 509 patients in comparison with whole tumor sections.

The multiple quantitative fluorescence staining of OPN, PD-L1 and TAMs was performed with monoclonal anti-rabbit OPN antibody at 1:200 dilution(Abcam, clone ab8448), PD-L1 antibody at 1:100 dilution(CST, clone E1L3N) and CD68 antibody at 1:100 dilution (Santa Cruz, clone ED1). Multiple quantitative fluorescence staining was performed with the Opal 7-Color Manual IHC Kit (NEL811001KT, PerkinElmer Inc. USA) according to the protocol of the manufacturer. Nuclei were stained with 4'-6'diamidino-2-phenylindole (DAPI, Thermo Scientific) after all the human antigens had been labelled. Negative control slides with the primary antibodies omitted were included in all assays. The staining slides were scanned by the Vectra System (PerkinElmer) to obtain multispectral images.

\section{Cell cultures}

HEK 293T cells, human NSCLC cells (H520 and A549), and mouse macrophage RAW264.7 cells were purchased from the Institute of Biochemistry and Cell Biology, Chinese Academy of Science (Shanghai China). These cells were cultured in DMEM or RPMI- 1640 medium supplemented with $10 \%$ fetal bovine serum (Gibco, Vienna, Austria). The cell lines were used for experiments within ten passages after thawing.

\section{Real-time PCR}

Total RNA was extracted from cultured cells, and reverse transcription and real-time PCR were performed as previously described ${ }^{23}$. For quantification of mRNA levels, GAPDH level was used as an internal control. Primer sequences for specific genes are shown below in Supplementary Table 1.

\section{Western blotting}

After separation by SDS-PAGE, the protein was transferred to a PVDF membrane, and blocked with milk in TBST and then incubated with primary antibodies against rabbit anti-PD-L1 (CST, clone E1L3N), p65 
(Abcam, clone ab32536) or a rabbit anti-phospho-NF-kB p65(Ser536) (Abcam, clone ab86299). HRPconjugated anti-rabbit secondary antibody (Santa Cruz Biotechnology, USA) was used as secondary antibodies.

\section{Cytokine and Inhibition of the NF-KB treatments}

NSCLC cells were incubated with of recombinant human OPN $(100 \mathrm{ng} / \mathrm{mL}, 200 \mathrm{ng} / \mathrm{mL}, 400 \mathrm{ng} / \mathrm{mL})(\mathrm{R} \& D$ Systems) at $37^{\circ} \mathrm{C}$ for 120 minutes. NSCLC cells were treated with Helenalin (Abcam, ab146197, 10 $\mathrm{ng} / \mathrm{mL}$ ) for 1 hour.

\section{Immunofluorescence}

For immunocytochemistry, cells grown on coverslips were fixed with $4 \%$ paraformaldehyde for 15 min and permeabilised with $0.1 \%$ Triton X-100 for 10 min. Cells were then incubated with $3 \%$ bovine serum albumin in PBS for 30 min to block the nonspecific binding sites. Cells were incubated overnight with rabbit polyclonal anti-p65 primary antibody (Abcam, clone ab32536, 1:50). After washing, cells were stained with a matching Alexa Fluor 488- conjugated secondary antibody (Life Technologies) at RT for 1 h, followed by DAPI staining (Invitrogen). Signals were visualized using a Confocal Laser Scanning Microscope.

\section{Animal studies}

Cell line-derived xenograft (CDX) models. Ten female nude mice were randomized into two groups(RAW264.7-OPN/A549, RAW-264.7-CtI/A549)(5 in each group, female BALB/c, 4-6weeks). A co-culture model of macrophages and lung cancer cells in vivo was constructed. Suspensions of $5 \times 10^{6}$ cells A549 and $1 \times 10^{6}$ cells RAW264.7 were injected subcutaneously into the flanks of mice obtained from the Model Animal Research Center of Nanjing University (Nanjing, China). Tumor volume was measured weekly using vernier caliper. The mice were sacrificed, and the tumor tissue was obtained at 5 weeks postimplantation. Consecutive sections were made for every tissue block of the tumor tissue and stained with hematoxylineosin.

\section{Statistical Analysis}

The association between variables was analyzed by $\chi 2$ testing analysis. Quantitation of TOPN and PD-L1 analyses was performed using Bonferroni correction. Overall survival was defined as the interval between the date of surgery and date of death or last follow-up. Disease-free survival was defined as the duration between the date of surgery and the date of first recurrence or last follow-up. OS and DFS were analyzed using the Kaplan-Meier method and compared using the log-rank test, and multivariate analysis were 
tested by Cox proportional hazard model. SPSS (version 22.0; IBM Corp, Armonk, NY) statistical programs for all analyses.

\section{Results}

\section{Clinical Characteristics}

A retrospective series of 509 NSCLC patients (296 males and 213 females, median age was 60 years, range 36 78) was retrieved from the original files of the Tianjin Cancer Institute and Hospital for the study. Patients' characteristics are described in Supplementary Table 2. Follow-up data were obtained from hospital charts and corresponding with the referring physicians. In this population, the mean followup was 47 months (ranging from 2-187 months), median OS[95\% confidence interval(Cl)] was 36(30.841.2) months.

\section{The Relationship Between Osteopontin Expression of Tumor-Associated Macrophages and PD-L1 Expression of NSCLC and Clinicopathologic Data}

The multiplex staining confirmed that OPN-positive macrophages occurred in $49.1 \%$ ( 250 of the 509 NSCLC patients) (Fig1A). And Of 509 NSCLC patients, there were 240 patients with high PD-L1 expression (Fig1). To validate the concordance between TMA and whole tumor sections, we further detected OPN, PD-L1, and TAMs for 50 cases randomly chosen from the 509 patients in comparison with whole tumor sections. We found that TAMs, PD-L1, and OPN expressed by TAMs(TOPN) in the whole tumor sections were $100 \%$ (50 of 50 ) in accordance with the results in the TMAs. To elucidate the biologic significance, we investigated the association of clinicopathologic features and PD-L1. As shown in Supplementary Table 3, PD-L1 was associated with Recurrence, TNM stage, T stage, $\mathrm{N}$ stage, subcarinal lymph node status, number of involved nodes and number of involved nodal station ( $P<0.05$, Supplementary Table 3$)$. The multiplex staining results showed that the expression of neoplastic PD-L1 in tumor with positive TOPN is significantly higher than that in patients with negative TOPN, and revealed a positive association between TOPN and neoplastic PD-L1 expression( $r=0.4497, P<0.0001)$ (Supplementary Table 3, Fig 1).

\section{Neoplastic PD-L1 and TOPN were Both Significantly Associated with Poor Prognosis in NSCLC Patients.}

The 5-year》8-year, 10-year overall survival rate was $39.10 \%, 35.17 \%$ and $34.77 \%$, the 5 -year $\$ 8$-year, 10 year disease free survival rate was $24.95 \%, 8.84 \%$ and $5.50 \%$ for the total study population. Analyzed by Kaplan-Meier log-rank test, both TOPN expression and PD-L1 were significant prognostic factors for NSCLC. (see online supplementary tables 4 and 5). Among overall 5-year $₫ 8$-year, 10-year survival rate and median survival time of NSCLC patients with higher PD-L1 expression were significantly lower than those 
in the group with lower PD-L1 expression(23.33\% vs. 53.16\%, 19.17\% vs. 49.44\%, 19.17\% vs. 48.70\%, 24 months vs. 55 months, $P<0.0001$,Fig $2 A$ ). The disease free 5 -year, 8 -year and 10 -year survival rate were $13.75 \%, 3.75 \%$ and $1.67 \%$ for PD-L1-higer group, $34.94 \% 13.75 \%$ and $8.92 \%$ for PD-L1-lower group, and median survival times were 17 months and 45 months for each subgroup $(P<0.0001 ;$ Fig $2 \mathrm{~B})$. Meanwhile, both overall 5-year, 8-year and 10-year survival rate and median survival time in TOPN+ group were significantly lower than those in the TOPN- group ( $16.40 \%$ vs. $61.00 \%, 13.20 \%$ vs. $56.37 \%, 12.80 \%$ vs. $55.60 \%, 24$ months vs. 60 months, $P<0.0001$; Fig $2 \mathrm{C}$ ). The disease free 5 -year, 8 -year and 10 year survival rate was $7.20 \%, 1.60 \%$ and $0.80 \%$ for TOPN+ group, $42.08 \%, 15.83 \%$, and $10.04 \%$ and for TOPNgroup $(P<0.0001 ;$ Fig 2D). Multivariate analysis indicated that overexpression of PD-L1 was an independent risk factor for both OS and DFS (Supplementary Table 6). These findings suggest PD-L1 and TOPN have the potential to serve as prognostic indicators for patients with NSCLC.

\section{PD-L1 Expression in NSCLC Cells is Enhanced after Co- cultured with Macrophages}

To further validate the association between OPN expressed by TAMs and neoplastic PD-L1, we first established an in vitro co-culture system by culturing NSCLC cells with different OPN expression levels RAW264.7 macrophage cells(Fig 3A). Then, we assessed the PD-L1 expressed by NSCLC cells. After coculture with macrophages, neoplastic PD-L1 expressed, which was co-cultured with RAW264.7-OPN, is significantly higher than the RAW264.7-CtI group(Fig 3B-3E). However, we did not observe significant changes in PD-L1 expression in OPN overexpression group or in OPN silence expression group of NSCLC cells(A549 and H520, Supplementary Fig 1). The results showed above strongly suggested that OPN expressed by TAMs in tumor microenvironment may influence PD-L1 expression via macrophages.

\section{OPN induces of PD-L1 expression in NSCLC cells via the NF-kB/p65 pathway.}

Treated NSCLC cells with recombinant human OPN, we found that OPN significantly increased PD-L1 expression both mRNA levels and protein expression in a dose-dependent manner (Fig 4A, Fig 4B, $P<0.001)$. Since it was known that OPN can activate NF-KB/p65 pathway, so we hypothesized that OPN might mediate PD-L1 upregulation through NF-kB/p65 pathway. To test this hypothesis, NF-kB/p65 pathway inhibitor, Helenalin, was used to block NF-kB/p65 pathway. Western blot and RT quantitative PCR showed that Helenalin could reverse the up-regulation of OPN on PD-L1(Fig 4C, Fig 4D P<0.001).

To further examine the effect of OPN on p65 subunit of NF-KB, both $\mathrm{A} 549$ and $\mathrm{H} 520$ cells were treated with $200 \mathrm{ng} / \mathrm{mL}$ recombinant human OPN and Helenalin in medium at $37^{\circ} \mathrm{C}$ for 0 to 120 minutes. And the results showed that after treated with recombinant human OPN, translocation of p 65 from cytoplasm to nucleus was observed (Fig 5A, Fig C). We examined the nuclear expression of p-p65 by western blot and 
found that p65 had cytoplasmic transfer to the nucleus, while p65 had no quantitative change (Fig 5B, Fig 5D).

After co-culture with macrophages, PD-L1 expressed by NSCLC cells(both A549 and H520) co-cultured with macrophages was significantly higher in RAW264.7-OPN group than that in the RAW264.7-CtI group(Fig 3). After the application of NF-KB inhibitor, it was showed that Helenalin could reverse the upregulation of neoplastic PD-L1 induced by TOPN(Fig 3A and 3B). Similarly, the addition of OPN antibody can reverse the up-regulation effect of TOPN on PD-L1(Fig 3C and 3D).

\section{Effect of TOPN on In Vivo Tumor Growth and PD-L1 Expression of NSCLC Cells}

It can be seen from the results in vivo that RAW-264.7-OPN/A549 co-coculture group had much larger histological lesions, compared with that of the RAW-264.7-Ctl/A549 co-culture group(Fig 6A, 6B) $\left(3817 \pm 311.4 \mathrm{~mm}^{3}\right.$ vs. $\left.1497 \pm 54.98 \mathrm{~mm}^{3}, P<0.001\right)$. Detected by the multiplex staining, the results showed that after co-cultured with macrophages in vivo, the NSCLC cells A549, which were co-cultured with RAW264.7-OPN, exhibited an increase in the level of PD-L1(Fig 6C) than the A549/RAW-264.7-Ctl co-cultured group(Fig 6D). The mRNA levels of several known antitumour immune mediators indicative, such as interferon (IFN- $\gamma$ ), tumour necrosis factor a (TNF-a) and C-X-C motif chemokine ligand 10 (CXCL10) were markedly reduced in RAW-264.7-OPN/A549 group. Simultaneously, we also observed a reduction in the expression IL-10, CSF1, and CXCL1, which were associated with M2 macrophage polarization(Fig 6E).

\section{Discussion}

Lung cancer remained the leading cause of cancer death. Although enormous improvement had occurred in clinical treatment strategies including immunotherapy, the prognosis is still unsatisfactory. PD-1/PD-L1 immune checkpoint blockade only provide durable responses from it in approximately $20 \%$ of patients ${ }^{10}$, 24 . As one of the two receptors of PD-1, PD-L1 plays an important role in the efficacy prognosis and immunosuppression. Tumor microenvironment is a crucial part of tumor for immunosuppression and tumor progression. The infiltration immune cells, such as TAMs ${ }^{24,25}$, produced immune suppressive factors. The urgent task is to clarify the regulatory effect of TAMs on PD-L1, and to find new therapy, which is combined with immunotherapy. So as to improve the long-term effect of patients, we can take timely intervention to avoid it.

Previously, Chen et al. ${ }^{26}$ reported that, examined by IHC, positive PD-L1 expression in primary cancer cells was found in $136(65.3 \%)$ patients, which were negatively correlated with lymph node metastasis $(P=0.009)$. And the results were similar with the Velcheti's ${ }^{27}$. However, in our study, PD-L1 was an independent prognostic factor of both OS and DFS in NSCLC patients $(P<0.001, P=0.024)$. It indicated that PD-L1 was positively associated with recurrence and metastasis, and TNM stage $(P<0.05)$. 
In immune microenvironment, TAM is the major source of the inflammatory cytokines ${ }^{28,29}$. Depending upon their microenvironment, TAMs mainly polarize toward "M1", which secret TNF-a, IL-12, and promote anti-tumor resistance, or "M2" phenotype that secret IL-10, TGF- $\beta$, play vital role in angiogenesis and tumor progression ${ }^{30}$. OPN is a phosphorylated glycoprotein that promotes cell adhesion, cell migratory activity, bone metabolism, and stone formation. Moreover, OPN, a prominent tumor-sustaining inflammatory mediator, is expressed not only by various malignant tumors but also by macrophages, and is also associated with tumor metastasis, proliferation and immunosuppression ${ }^{31}$. Previous study by our group ${ }^{18}$ show that TAMs inflitration was not an independent prognostic factor of NSCLC, and has no effect on tumor metastasis. However, TOPN plays an important role in tumor metastasis of NSCLC and is an independent prognostic factor for NSCLC patients. Wei et $\mathrm{al}^{32}$. repoted that, in glioblastoma, OPN maintained the gene characteristics and phenotype of M2 macrophages. OPN plays a critical role in the development of glioblastoma. OPN deficiency innated immune, and resulted in a marked decrease in M2 macrophages and an increase in T cell effector activity in gliomas. It was reported that overexpression of PD-L1 may be induced by macrophages in HCC and PDAC 23,33 . In the current study, application of multiple quantitative fluorescence staining detection of a clinical NSCLC data set the resulted showed that TOPN, but not TAMs was positively correlated with the expression of PD-L1 in NSCLC patients. It can be concluded that not all TAMs have regulatory effect on PD-L1, and the expression of PD-L1 in tumor cells can be promoted by TOPN.

Previous studies have shown that the expression of PD-L1 in tumor cells can be regulated by many factors and pathways in the microenvironment. As for non-immune cells, PD-L1 can be induced by IFN- $\gamma$ and TNF-a on endothelial cells ${ }^{34}$. Another research reported by Chen et al. ${ }^{33}$ showed that the macrophages induced upregulation of PD-L1 expression in HCC cells through NF-KB and STAT3 pathways and suggested that the overexpression of PD-L1 in HCC may be mediated by inflammatory microenvironment involving macrophages. Likewise, PD-L1 expression on PDAC cells was also positively related with macrophage infltration in tumor stroma and infiltrating macrophages derived TNF-a upregulated PD-L1 expression on PDAC cells via NF-KB pathway ${ }^{23}$. Lim et al ${ }^{35}$ showed that COP9 signalosome 5 (CSN5), induced by NF-kB p65, is required for TNF-a-mediated PD-L1 stabilization in breast cancer cells.

In this study, we uncovered the correlation between TOPN and upregulation of immune checkpoint PD-L1 as a potential mechanism by which NSCLC escapes immune surveillance. Our findings provided further evidence that OPN significantly increased PD-L1 expression both mRNA levels and protein expression in a dose-dependent manner. In vitro co-culture system, PD-L1 expressed by NSCLC cells co-cultured with macrophages was significantly higher in RAW264.7-OPN group than that in the RAW264.7-Ctl group. Both NF-KB pathway inhibitor and OPN antibody has the potential to reverse the up-regulation of PD-L1 induced by TOPN in NSCLC. Moreover, there were not significant changes in PD-L1 expression in OPN overexpression group or in OPN silence expression group of NSCLC cells. The results showed above strongly suggested that OPN expressed by TAMs in tumor microenvironment may influence PD-L1 expression via macrophages. As M2 macrophages, they secrete IL-10, CSF1, and CXCL1, which play a 
role in angiogenesis, tumor progression, and immunosuppression ${ }^{30}$. In nude mice model, TOPN also can promote the growth of tumor cells and induce the expression of PD-L1 of NSCLC. In addition, in the RAW264.7-OPN group, the mRNA level of tumor suppressor immunostimulatory factors decreased, while the level of tumor immunosuppressive factors increased, indicating that TOPN could promote tumor in microenvironment. OPN could be a marker for define M2 TAMs, and could be a new unfavorable factor to discriminate biological functions of TAMs. In conclusion, TOPN could induce PD-L1 expression via NF-KB pathway and promoted tumor growth in NSCLC.

The next frontier of immunotherapy is to find ways to improve the efficacy of checkpoint inhibitors through joint approaches, especially in cancer with limited clinical efficacy. Recent studies have shown that the combination of anti-PD-1/PD-L1 and CSF-1R inhibitor ${ }^{36}$, TGF- $\beta$ inhibitor ${ }^{37}$ and blocking FcyR on macrophage ${ }^{38}$ can synergistically enhance anti-tumor immunity. In view of the important role of TOPN in tumor progression, metastasis, immunosuppression and regulation of PD-L1, the treatment methods for TOPN need to be developed urgently in order to achieve better effect in combination with anti-PD-1/PDL1.

In conclusion, PD-L1 and TOPN would be unfavorable prognostic factors for NSCLC patients, who underwent curative resection. And TOPN induced Tumor-mediated secretion PD-L1 via NF-kB/p65 pathway. Thus, timely treatment, for NSCLC patients with TOPN+ or PD-L1+, should be undertaken to reduce tumor metastasis and prolong survival. Moreover, TOPN may be a new factor to discriminate biological functions of TAMs. The combination of blocking OPN on macrophage and antiPD-1/PD-L1 might shape the future of cancer immunotherapy.

\section{Conclusions}

Overall, We investigate the relationship between the OPN expression in TAMs (TOPN) and Neoplastic PDL1 in completely resected NSCLC patients and the regulatory mechanism of TOPN on PD-L1. We revealed that TOPN-NF-KB axis plays a critical role in PD-L1 expression of NSCLC microenvironment and TOPN could be a potential therapeutic target for NSCLC.

\section{Abbreviations}

TAMs: Tumor-associated macrophages

OPN: Osteopontin

TOPN: OPN expressed by TAMs

OS: overall survival

DFS: disease-free survival

NSCLC: Non-small cell lung cancer 
FDA: the US Food and Drug Administration

mAbs: Monoclonal antibodies

RCC: renal cell carcinoma

CDX: Cell line-derived xenograft

Cl: confidence interval

\section{Declarations}

\section{Ethics approval and consent to participate}

This study was approved by the research ethics committee of Tianjin Cancer Institute and Hospital, and all patients involved provided written informed consent.

\section{Consent for publication}

All authors have given consent for publication.

\section{Availability of data and materials}

All data generated or analyzed during this study are included in this published article (and its supplementary information files).

\section{Competing interests}

The authors declare that they have no competing interests.

\section{Funding}

This work was supported by National Natural Science Foundation of China『No. 81772484, No.81772488区

\section{Authors' contributions}

All authors have contributed significantly, and that all authors read and approved the final manuscript.

\section{Acknowledgments}

None

\section{Reference}


1. Petrelli NJ, Winer EP, Brahmer J, et al. Clinical Cancer Advances 2009: major research advances in cancer treatment, prevention, and screening-a report from the American Society of Clinical Oncology. J Clin Oncol 2009;27:6052-6069.

2. Mountain CF. Revisions in the International System for Staging Lung Cancer. Chest 1997;111:17101717.

3. Varlotto JM, Recht A, Flickinger JC, et al. Factors associated with local and distant recurrence and survival in patients with resected nonsmall cell lung cancer. Cancer 2009;115:1059-1069.

4. Goldstraw P, Chansky K, Crowley J, et al. The IASLC Lung Cancer Staging Project: Proposals for Revision of the TNM Stage Groupings in the Forthcoming (Eighth) Edition of the TNM Classification for Lung Cancer. J Thorac Oncol 2016;11:39-51.

5. Jemal A, Siegel R, Ward E, et al. Cancer statistics, 2009. CA Cancer J Clin 2009;59:225-249.

6. Diker O. Atezolizumab plus Chemotherapy in Small-Cell Lung Cancer. N Engl J Med 2019;380:889.

7. Gandhi L, Rodriguez-Abreu D, Gadgeel S, et al. Pembrolizumab plus Chemotherapy in Metastatic Non-Small-Cell Lung Cancer. N Engl J Med 2018;378:2078-2092.

8. Rittmeyer A, Barlesi F, Waterkamp D, et al. Atezolizumab versus docetaxel in patients with previously treated non-small-cell lung cancer (OAK): a phase 3, open-label, multicentre randomised controlled trial. Lancet 2017;389:255-265.

9. Sharpe AH, Pauken KE. The diverse functions of the PD1 inhibitory pathway. Nat Rev Immunol 2018;18:153-167.

10. Page DB, Postow MA, Callahan MK, et al. Immune modulation in cancer with antibodies. Annu Rev Med 2014;65:185-202.

11. Goldberg MV, Maris $\mathrm{CH}$, Hipkiss EL, et al. Role of PD-1 and its ligand, $\mathrm{B} 7-\mathrm{H} 1$, in early fate decisions of CD8 T cells. Blood 2007;110:186-192.

12. Chen $\mathrm{YB}, \mathrm{Mu} \mathrm{CY}$, Huang JA. Clinical significance of programmed death-1 ligand-1 expression in patients with non-small cell lung cancer: a 5-year-follow-up study. Tumori 2012;98:751-755.

13. Mu CY, Huang JA, Chen Y, et al. High expression of PD-L1 in lung cancer may contribute to poor prognosis and tumor cells immune escape through suppressing tumor infiltrating dendritic cells maturation. Med Oncol 2011;28:682-688.

14. Kryczek I, Wei S, Zhu G, et al. Relationship between B7-H4, regulatory T cells, and patient outcome in human ovarian carcinoma. Cancer Res 2007;67:8900-8905.

15. Alderton GK. Tumour immunology: turning macrophages on, off and on again. Nat Rev Immunol 2014;14:136-137.

16. Noy R, Pollard JW. Tumor-associated macrophages: from mechanisms to therapy. Immunity 2014;41:49-61.

17. Rangaswami H, Bulbule A, Kundu GC. Osteopontin: role in cell signaling and cancer progression. Trends Cell Biol 2006;16:79-87. 
18. Li Y, Sun BS, Pei B, et al. Osteopontin-expressing macrophages in non-small cell lung cancer predict survival. Ann Thorac Surg 2015;99:1140-1148.

19. Sun BS, Dong QZ, Ye QH, et al. Lentiviral-mediated miRNA against osteopontin suppresses tumor growth and metastasis of human hepatocellular carcinoma. Hepatology 2008;48:1834-1842.

20. Sun BS, Li Y, Zhang ZF, et al. Osteopontin combined with CD44v6, a novel prognostic biomarker in non-small cell lung cancer undergoing curative resection. Ann Thorac Surg 2013;96:1943-1951.

21. Sun BS, You J, Li Y, et al. Osteopontin knockdown suppresses non-small cell lung cancer cell invasion and metastasis. Chin Med J (Engl) 2013;126:1683-1688.

22. Imano $M$, Okuno $K$, Itoh $T$, et al. Increased osteopontin-positive macrophage expression in colorectal cancer stroma with synchronous liver metastasis. World J Surg 2010;34:1930-1936.

23. Tsukamoto M, Imai K, Ishimoto T, et al. PD-L1 expression enhancement by infiltrating macrophagederived tumor necrosis factor-alpha leads to poor pancreatic cancer prognosis. Cancer Sci 2019;110:310-320.

24. Coussens LM, Werb Z. Inflammation and cancer. Nature 2002;420:860-867.

25. Pitt JM, Vetizou M, Daillere R, et al. Resistance Mechanisms to Immune-Checkpoint Blockade in Cancer: Tumor-Intrinsic and -Extrinsic Factors. Immunity 2016;44:1255-1269.

26. Chen YY, Wang LB, Zhu HL, et al. Relationship between programmed death-ligand 1 and clinicopathological characteristics in non-small cell lung cancer patients. Chin Med Sci J 2013;28:147-151.

27. Velcheti V, Schalper KA, Carvajal DE, et al. Programmed death ligand-1 expression in non-small cell lung cancer. Lab Invest 2014;94:107-116.

28. Mantovani A, Sica A. Macrophages, innate immunity and cancer: balance, tolerance, and diversity. Curr Opin Immunol 2010;22:231-237.

29. Grivennikov SI, Greten FR, Karin M. Immunity, inflammation, and cancer. Cell 2010;140:883-899.

30. Martinez FO, Gordon S. The M1 and M2 paradigm of macrophage activation: time for reassessment. F1000Prime Rep 2014;6:13.

31. Sangaletti S, Tripodo C, Sandri S, et al. Osteopontin shapes immunosuppression in the metastatic niche. Cancer Res 2014;74:4706-4719.

32. Wei J, Marisetty A, Schrand B, et al. Osteopontin mediates glioblastoma-associated macrophage infiltration and is a potential therapeutic target. $J$ Clin Invest 2019;129:137-149.

33. Chen J, Li G, Meng H, et al. Upregulation of B7-H1 expression is associated with macrophage infiltration in hepatocellular carcinomas. Cancer Immunol Immunother 2012;61:101-108.

34. Mazanet MM, Hughes CC. B7-H1 is expressed by human endothelial cells and suppresses $\mathrm{T}$ cell cytokine synthesis. J Immunol 2002;169:3581-3588.

35. Lim SO, Li CW, Xia W, et al. Deubiquitination and Stabilization of PD-L1 by CSN5. Cancer Cell 2016;30:925-939. 
36. Zhu Y, Yang J, Xu D, et al. Disruption of tumour-associated macrophage trafficking by the osteopontin-induced colony-stimulating factor-1 signalling sensitises hepatocellular carcinoma to anti-PD-L1 blockade. Gut 2019;68:1653-1666.

37. Mariathasan S, Turley SJ, Nickles D, et al. TGFbeta attenuates tumour response to PD-L1 blockade by contributing to exclusion of T cells. Nature 2018;554:544-548.

38. Arlauckas SP, Garris CS, Kohler RH, et al. In vivo imaging reveals a tumor-associated macrophagemediated resistance pathway in anti-PD-1 therapy. Sci Trans/ Med 2017;9.

\section{Tables}


Table 1

Relationship between PD-L1 and Clinicopathologic Factors of Patients

\begin{tabular}{|c|c|c|c|}
\hline & PD-L1 & & \\
\hline Factors & Low, n(\%) & High, n(\%) & $P$ value \\
\hline Gender & & & 0.0156 \\
\hline Male & $143(28.09 \%)$ & $153(30.06 \%)$ & \\
\hline Female & $126(24.75 \%)$ & $87(17.09 \%)$ & \\
\hline Age & & & 0.8760 \\
\hline$<60 y$ & 133(26.13\%) & $117(22.99 \%)$ & \\
\hline$\geq 60 y$ & $136(26.72 \%)$ & $123(24.17 \%)$ & \\
\hline Recurrence & & & $<0.0001$ \\
\hline No & $114(22.40 \%)$ & $54(10.61 \%)$ & \\
\hline Yes & $155(30.45 \%)$ & $186(36.54 \%)$ & \\
\hline Smoking status & & & 0.0023 \\
\hline Never smoke & $129(25.34 \%)$ & $83(16.31 \%)$ & \\
\hline Smoke & $140(27.50 \%)$ & $157(30.84 \%)$ & \\
\hline Type of resection & & & 0.9502 \\
\hline Lobectomy & $219(43.03 \%)$ & 198(38.90\%) & \\
\hline Pneumonectomy & $32(6.29 \%)$ & $27(5.30 \%)$ & \\
\hline Bronchial sleeve resection & $18(3.54 \%)$ & $15(2.95 \%)$ & \\
\hline Lesion & & & 0.7667 \\
\hline Central & $62(12.18 \%)$ & $58(11.39 \%)$ & \\
\hline Peripheral & $207(40.67 \%)$ & $182(35.76 \%)$ & \\
\hline Location of tumor & & & 0.5711 \\
\hline Left & $111(21.81 \%)$ & $105(20.63 \%)$ & \\
\hline Right & 158(31.04\%) & $135(26.52 \%)$ & \\
\hline Histologic subtype & & & 0.0097 \\
\hline Squamous cell carcinoma & $68(13.36 \%)$ & $86(16.90 \%)$ & \\
\hline Adenocarcinoma & 201(39.49\%) & $154(30.26 \%)$ & \\
\hline Stage & & & 0.0016 \\
\hline
\end{tabular}




\begin{tabular}{|c|c|c|c|}
\hline I & $94(18.47 \%)$ & $50(9.82 \%)$ & \\
\hline II & $54(10.61 \%)$ & $53(10.41 \%)$ & \\
\hline IIIA & $121(23.77 \%)$ & $137(26.92 \%)$ & \\
\hline T stage & & & 0.0022 \\
\hline T1 & 123(24.17\%) & $81(15.91 \%)$ & \\
\hline T2 & 99(19.45\%) & $85(16.70 \%)$ & \\
\hline T3 & $29(5.70 \%)$ & $50(9.82 \%)$ & \\
\hline T4 & $18(3.54 \%)$ & $24(4.72 \%)$ & \\
\hline N stage & & & 0.0094 \\
\hline NO & $129(25.34 \%)$ & $83(16.31 \%)$ & \\
\hline N1 & $31(6.09 \%)$ & $34(6.68 \%)$ & \\
\hline N2 & $109(21.41 \%)$ & $123(24.17 \%)$ & \\
\hline Subcarinal lymph node & & & 0.0009 \\
\hline Negative & $222(43.61 \%)$ & 168(33.01\%) & \\
\hline Positive & $47(9.23 \%)$ & 72(14.15\%) & \\
\hline Number of involved nodes & & & 0.0005 \\
\hline$\leq 4$ & $208(40.86 \%)$ & 152(29.86\%) & \\
\hline$>4$ & $61(11.98 \%)$ & $88(17.29 \%)$ & \\
\hline Number of involved nodal station & & & 0.0024 \\
\hline$\otimes 2$ & 179(35.17\%) & $128(25.15 \%)$ & \\
\hline$\geq 2$ & $90(17.68 \%)$ & $112(22.00 \%)$ & \\
\hline TOPN & & & $<0.0001$ \\
\hline Negative & 194(38.11\%) & $65(12.77 \%)$ & \\
\hline Positive & $75(14.73 \%)$ & 175(34.38\%) & \\
\hline TAMs & & & 0.2067 \\
\hline Negative & 119(23.38\%) & 101(19.84\%) & \\
\hline Positive & $140(27.50 \%)$ & 149(29.27\%) & \\
\hline
\end{tabular}

Abbreviations: TAMs, Tumor-associated macrophages; TOPN, OPN expressed by TAMs; TNM, tumornode-metastasis; 
Table 2

Prognostic Factors for DFS Retained Multivariate Analysis in 509 NSCLC Patients

\begin{tabular}{|c|c|c|c|c|}
\hline & OS & & DFS & \\
\hline Variables & $H R \quad(95.0 \% \mathrm{Cl})$ & $P$ & $95.0 \% \mathrm{Cl}$ & $P$ \\
\hline TNM & $1.114(1.012 \sim 1.226)$ & 0.027 & 1.368(1.021 1.832) & 0.036 \\
\hline T stage & $0.765(0.519 \sim 1.128)$ & 0.176 & 1.072(0.972 1.183) & 0.166 \\
\hline $\mathrm{N}$ stage & $1.623(1.119 \sim 2.254)$ & 0.011 & 1.494(1.037 2.154) & 0.031 \\
\hline Subcarinal lymph node & $1.264(0.947 \sim 1.689)$ & 0.112 & $0.817(0.0 .559 \sim 1.195)$ & 0.297 \\
\hline $\begin{array}{l}\text { Number of involved nodal } \\
\text { station }\end{array}$ & $1.445(0.976 \sim 2.140)$ & 0.066 & $1.415(0.946 \sim 2.118)$ & 0.091 \\
\hline Number of involved nodes & $0.918(0.656 \sim 1.285)$ & 0.619 & $0.972(0.689 \sim 1.371)$ & 0.873 \\
\hline TOPN & $2.732(2.110 \sim 3.537)$ & $<0.0001$ & $2.176(1.685 \sim 2.811)$ & $<0.0001$ \\
\hline PD-L1 & $1.495(1.172 \sim 1.908)$ & $<0.001$ & $1.330(1.038 \sim 1.703)$ & 0.024 \\
\hline
\end{tabular}

Abbreviations: TOPN, OPN expressed by TAMs; TNM, tumor-node-metastasis; OS, overall survival; DFS, disease free survival; $\mathrm{HR}$, hazard ration; $\mathrm{Cl}$, confidence interval

Figures 

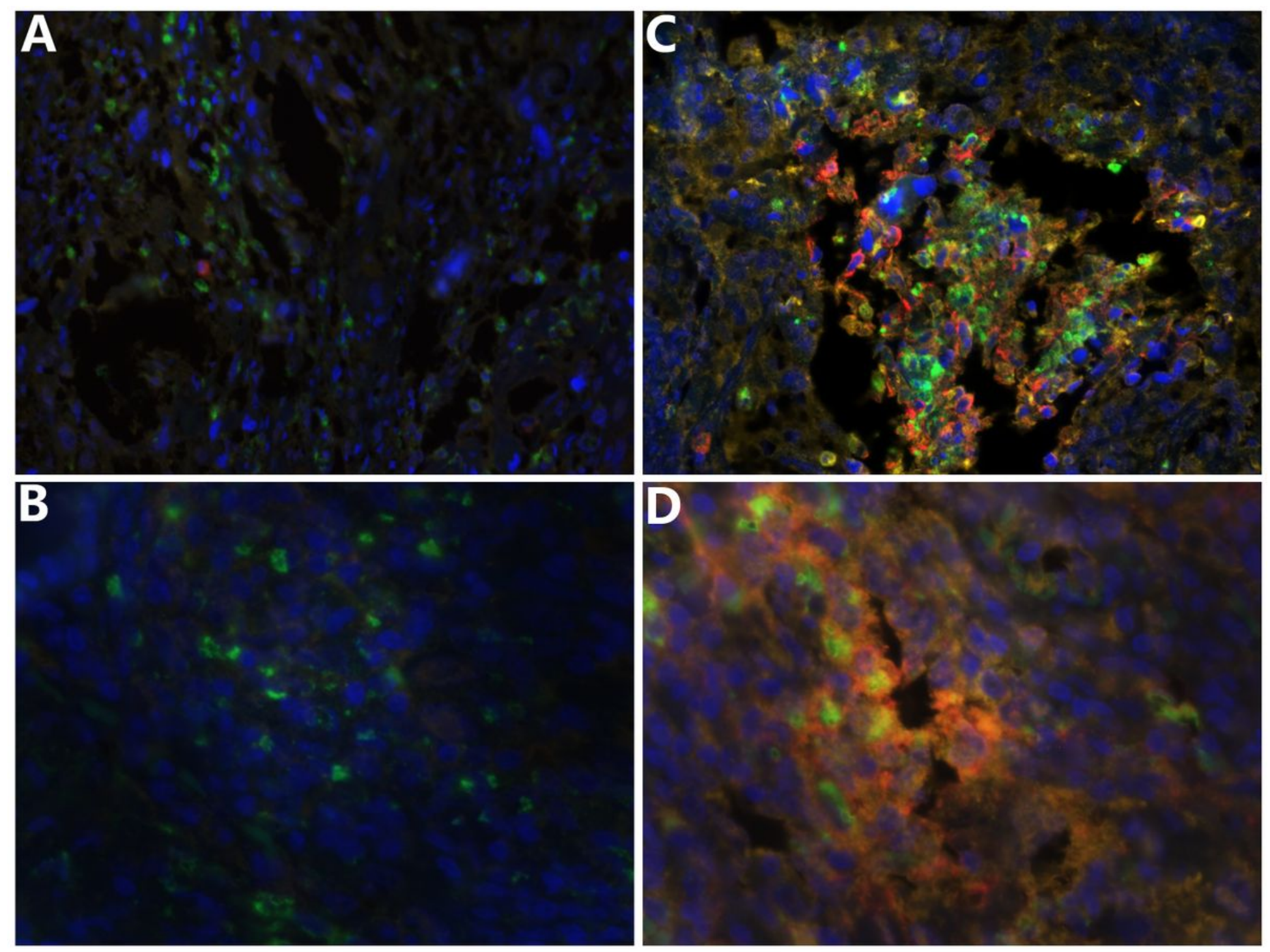

\section{Figure 1}

Multiple quantitative fluorescence staining. DAPI(blue), OPN(red), CD68(green) and PD-L1(yellow). OPN expressed by TAMs showed a significant positive correlation with the PD-L1 expression on NSCLC cells. (A) TOPN- with PD-L1low 20x;(B)TOPN- with PD-L1low 40x; (C) TOPN+ with PD-L1high 200x;(D)TOPN+ with PD-L1high 400x; 

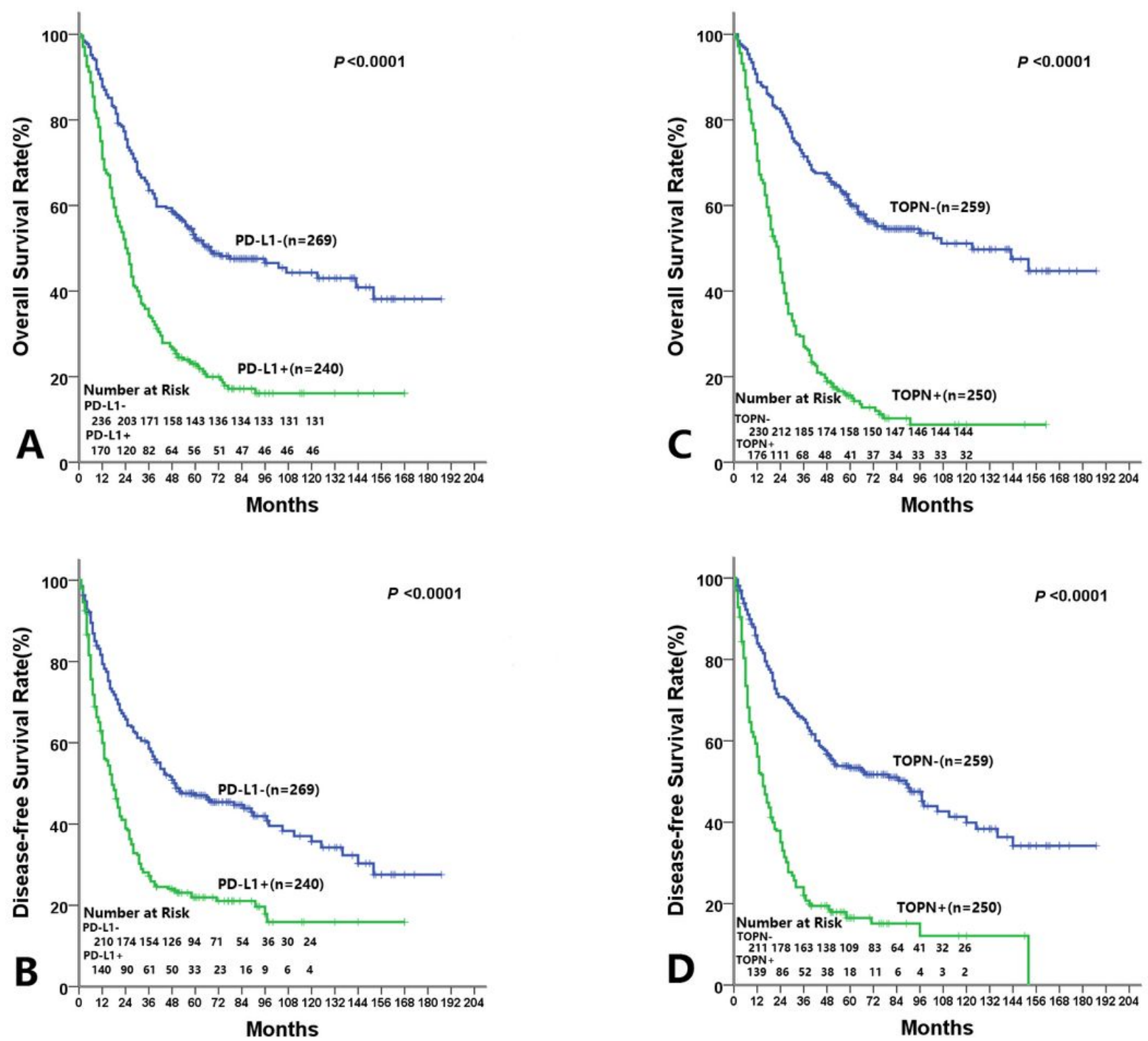

Figure 2

Prognostic significance assessed using Kaplan-Meier survival estimates and log-rank tests. Comparisons of OS and DFS by PD-L1(A, B) and TOPN(C, D) 


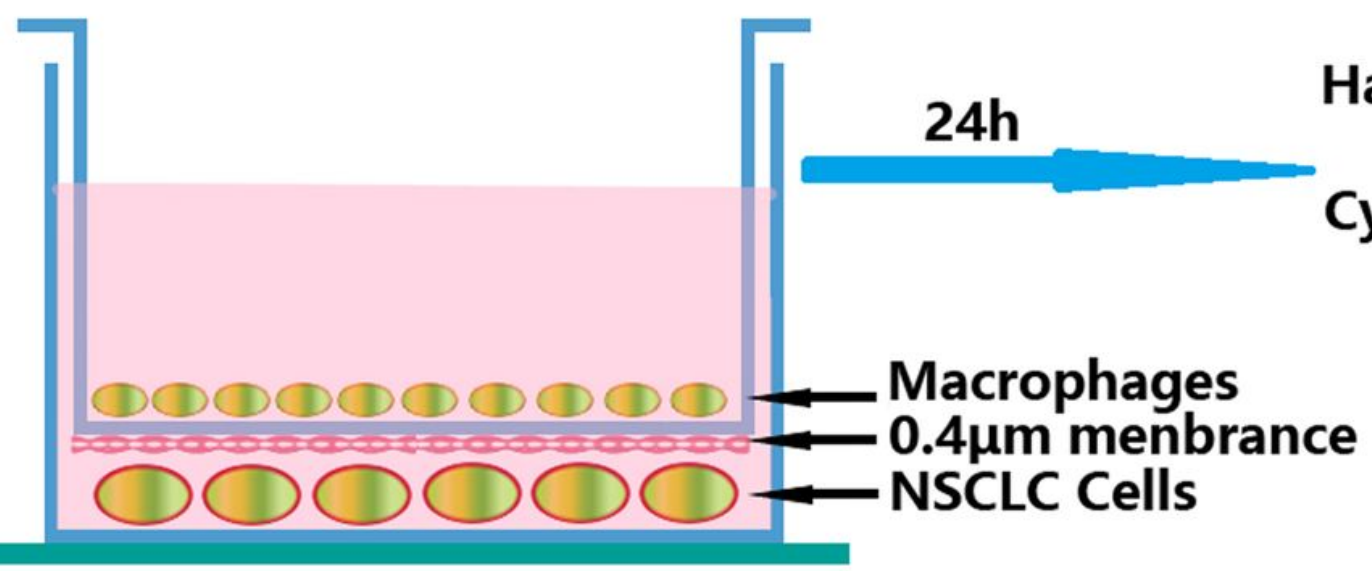

B A549 GAPDH PD-L1

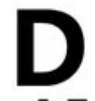
A549

\section{Harvest cells}

\section{Cytokine analysis}

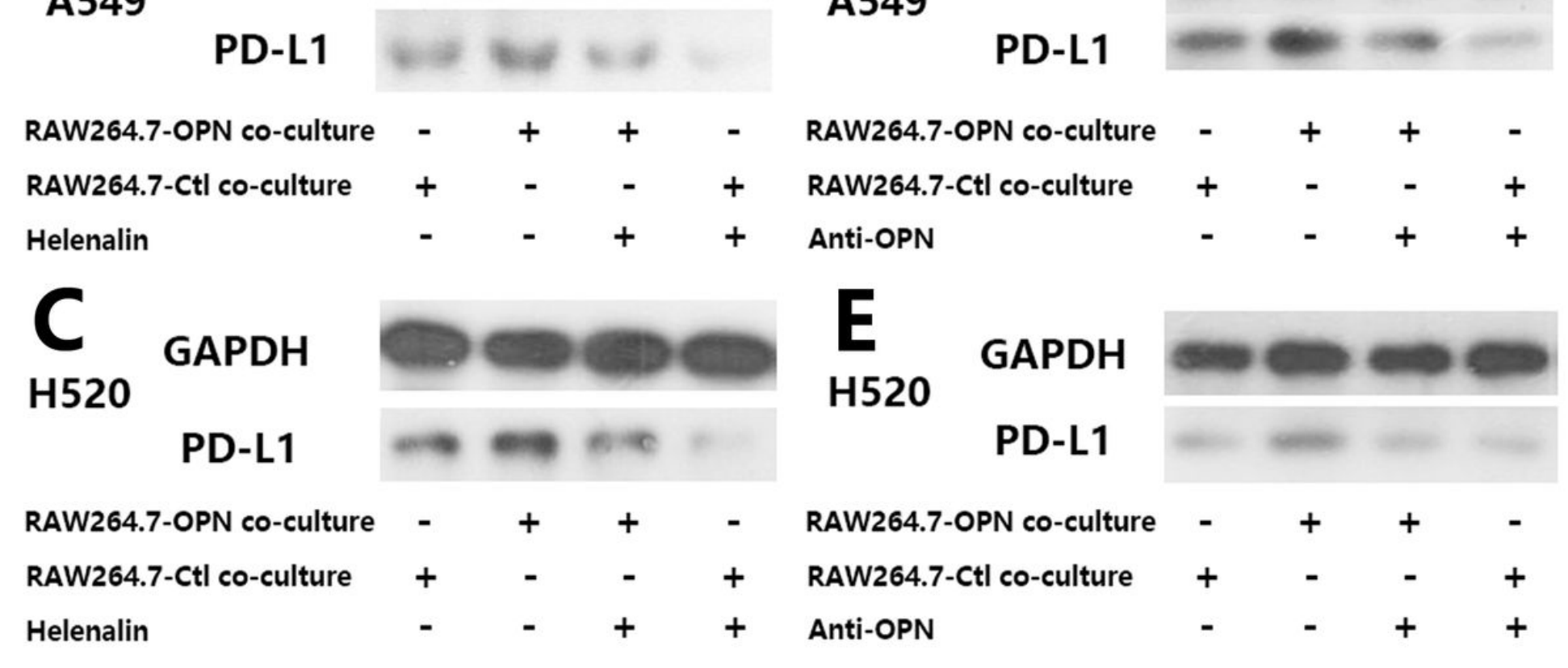

Figure 3

(A) Schematic showing the NSCLC cells cocultured with macrophages of different OPN levels in a transwell apparatus of $0.4 \mu \mathrm{m}$ pore size; (B,C,D and E) A549 and H520 cells were treated with Helenalin (Abcam, ab146197, $10 \mathrm{ng} / \mathrm{mL}$ ) for 1 hour or monoclonal anti-rabbit OPN antibody, and then were cocultured with macrophages (RAW264.7-Ctl or RAW264.7-OPN) for 24 hours. Western blot analysis of PD-L1 expression in different co-coculture groups. 

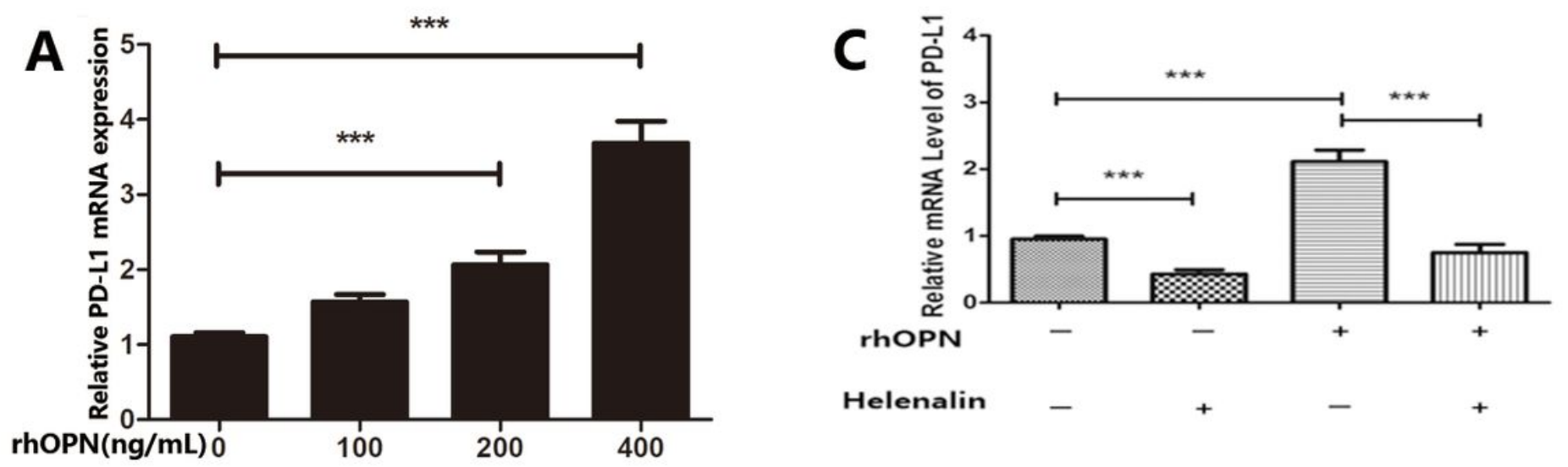

B

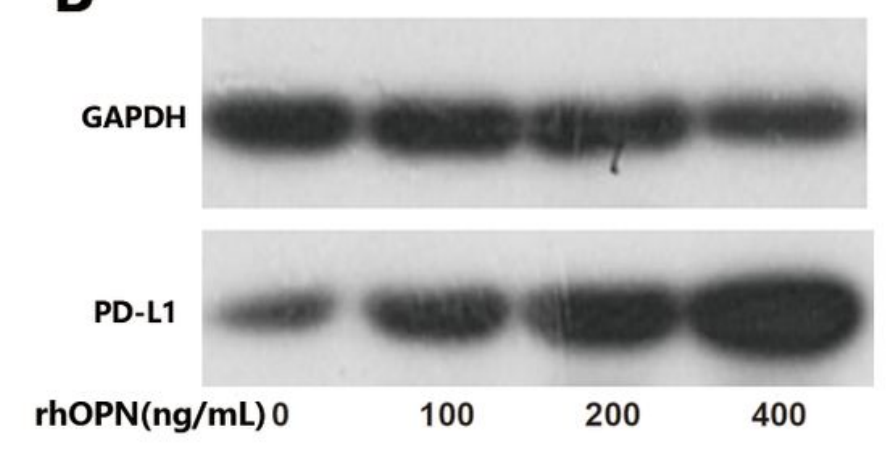

D

GAPDH

PD-L1

rhOPN

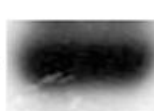

Helenalin

Figure 4

NSCLC cells(A549) were incubated with of recombinant human OPN(100ng/mL, $200 \mathrm{ng} / \mathrm{mL}, 400 \mathrm{ng} / \mathrm{mL}$ ) (R\&D Systems) at $37^{\circ} \mathrm{C}$ for 120 minutes. mRNA and protein were examined by RT-PCR(A) and Western blot(B); NSCLC cells were treated with Helenalin for 1 hour and then incubated with of recombinant human OPN(200 ng/mL), RT-PCR(C) and Western blot(D) were examined. 


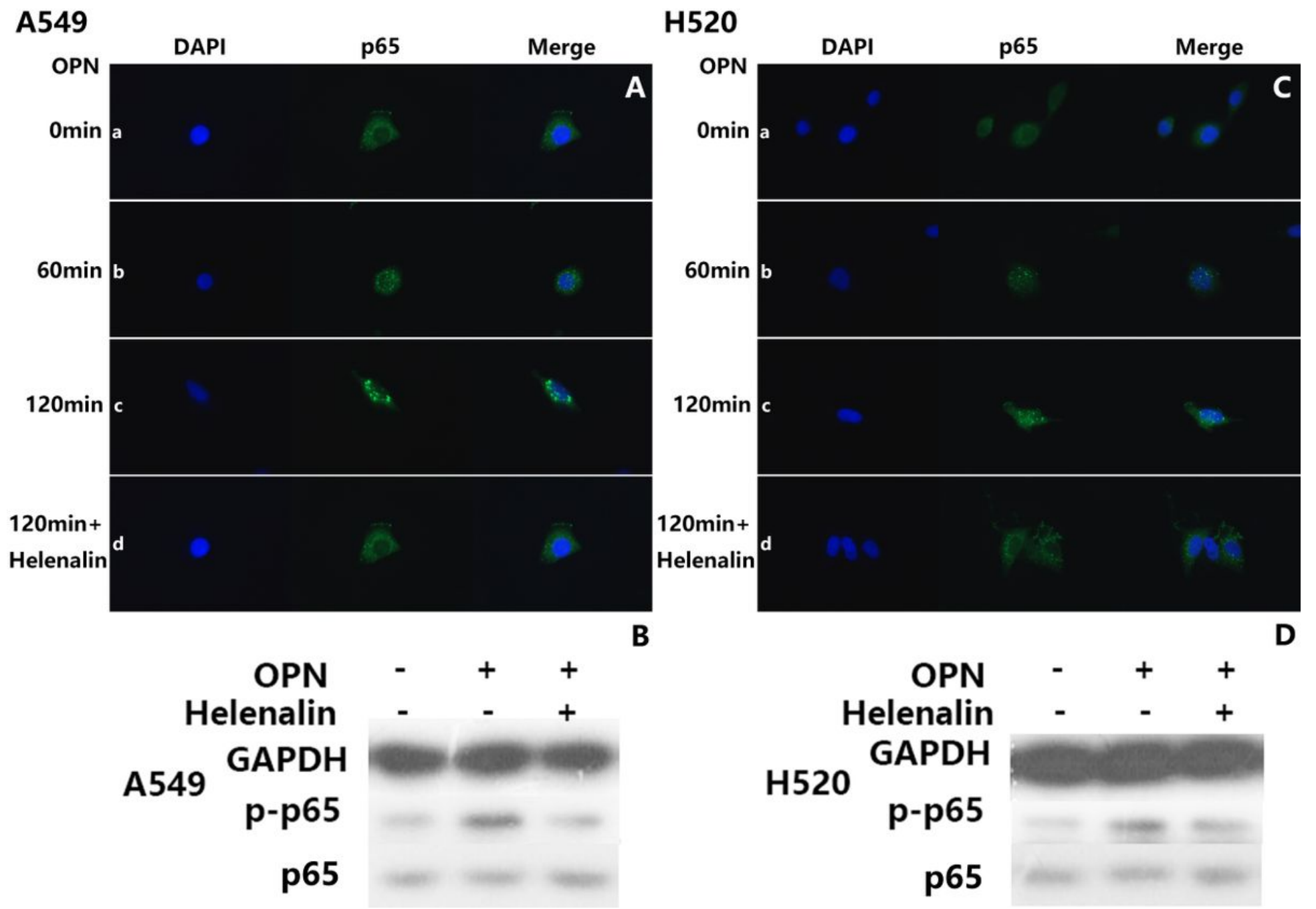

Figure 5

Immunofluorescence assay for the localization of p65 protein in A549(A) and H520 cells(C). A549 and H520 cells grown on glass slides were treated with $200 \mathrm{ng} / \mathrm{mL}$ of OPN (for 0-120 minutes). p65 was mainly located in cytoplasm of A549 and H520 cells before OPN treatment (a). From 60 minutes (b) and 120 minutes (c) after OPN treatment, translocation of p65 into the nucleus was observed and increased; p65 was completely accumulated in the nucleus of NSCLC cells after OPN treatment for 120 minutes (c). The addition of Helenalin can reverse the up-regulation effect of OPN on translocation of p65 into the nucleus(d). Western blot analysis of proteins detected by probing with anti-p65 or anti-phosphorylated p65 antibodies. Recombinant human OPN increased the level of phosphorylated p65(B, D). 
A

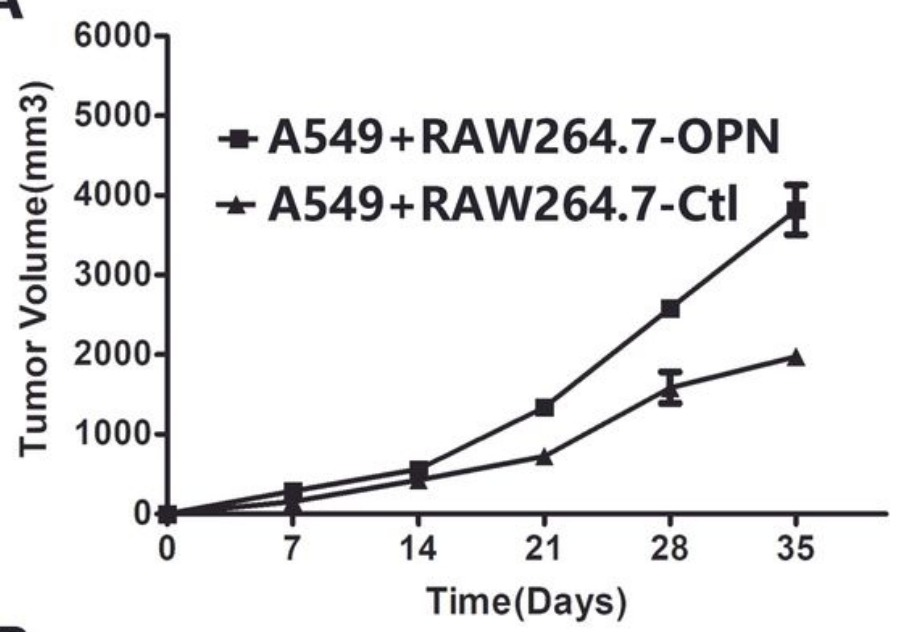

B

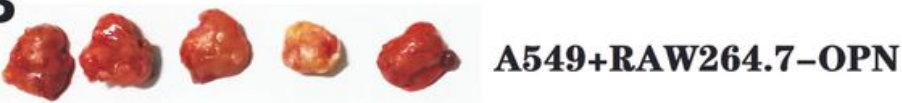

D

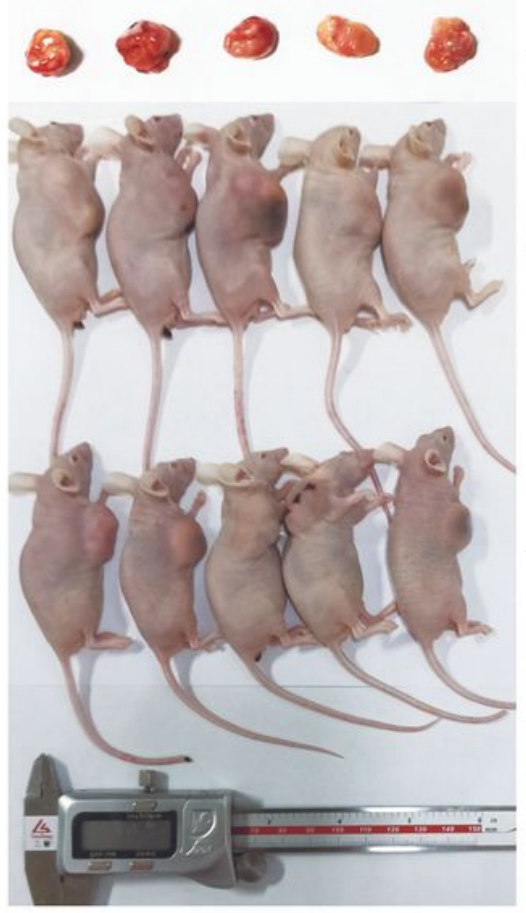

A549+RAW264.7-Ctl

A549+RAW264.7-OPN

A549+RAW264.7-Ctl

E

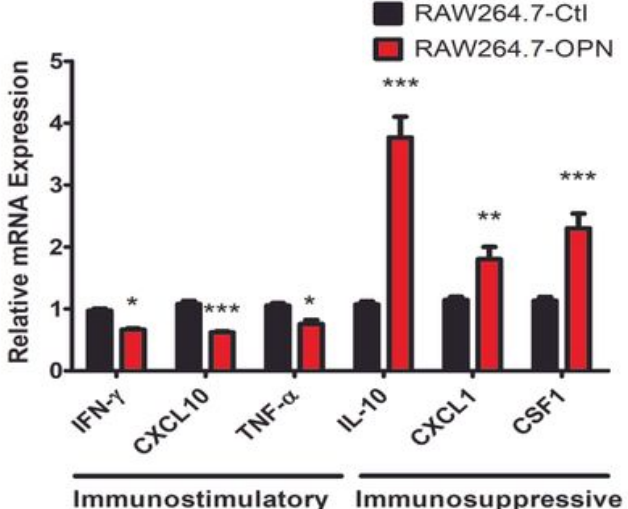

factors

factors

\section{Figure 6}

The effect of TOPN on the in vivo tumor growth and the PD-L1 expression of A549 cells. (A, B) In vivo tumor growth in RAW-264.7-OPN mice was significantly larger compared with the RAW-264.7-Ctl group in tumor volumes. The NSCLC cells A549, which were co-cultured with RAW-264.7-OPN, exhibited an increase in the level of PD-L1(C) than the A549/RAW-264.7-Ctl co-cultured group(D). Relative expression of the indicated genes by qPCR(E). Data depict the Mean $\pm \operatorname{SEM}(n=6)$. 


\section{Supplementary Files}

This is a list of supplementary files associated with this preprint. Click to download.

- SupplementaryFig1.jpg

- SupplementaryTable.docx 